\title{
Estrategias colaborativas y open-source para la generación de sistemas productivos entre diseñadores y productores
}

\author{
Gajardo Valdés, Rodrigo Francisco; Gerea Petculescu, Carmen \& Mollenhauer Gajardo, Katherine ${ }^{\mathrm{c}}$ \\ åontificia Universidad Católica de Chile, Universidad Tecnológica Metropolitana, Chile.rfgajardo@uc.cl \\ ${ }^{\mathrm{b}}$ Pontificia Universidad Católica de Chile, Chile. cgerea@uc.cl \\ cPontificia Universidad Católica de Chile, Chile. kamollenhauer@uc.cl
}

\begin{abstract}
:
In this paper, we explore the trend of return of the crafts, the emergence of new designer brands and local manufacturing derived from different disciplines and a consumer increasingly demanding in terms of product traceability and narrative. However, this movement of makers with local producers has his own problems, as the lack of consistent production systems for local economies. Therefore, the sustainability of these projects and initiatives might be in danger.

The collaboration between artisans or producers and designers has been the subject of work in various government and university initiatives. Consequently, there is a number of projects that have used different models of collaboration between these actors from a centralized perspective, to a closed model, or even an opened and experimental one. Models of interaction between the actors, their roles, results and sustainability of these projects will be established as the main field of study.
\end{abstract}

The aim of this paper is to analyze the different models of collaboration observes in three case studies of initiatives that have connected designers and artisans or producers. The methodology used for comparative analysis is the study of multiple case. The first two -Laboratory Design Colombia and Rush Weaving of Taiwanwere chosen for being documented and emblematic cases in the literature. The third -SaberHacer of Chile- is a work in progress project of one of the authors of this article.

This article aims to identify those elements in collaborative models that might impact the sustainability of the projects studied and could be set as inputs for the development and systematization of new work models as well as new scenarios that generate new research in this field of study.

Keywords: Productive systems, design, crafts, collaborative economy, study of cases. 
Estrategias colaborativas y open-source para la generación de sistemas productivos entre diseñadores y productores.

\section{Resumen}

En el siguiente artículo, se explorará en torno a la tendencia del retorno de los oficios, el surgimiento de nuevas marcas de diseño y manufactura local derivadas de distintas disciplinas y un consumidor cada vez más exigente en cuanto a la trazabilidad del producto, constituyéndose esta como una experiencia en sí misma. Sin embargo, la interrelación de este movimiento de creadores con productores locales ha dejado a la vista el problema de la inexistencia de sistemas productivos acordes a las economías locales que lo sustentan y por tanto el peligro de la sustentabilidad de estos mismos emprendimientos e iniciativas.

La colaboración entre artesanos o productores y diseñadores ha sido materia de trabajo en diversas iniciativas gubernamentales y universitarias, encontrándonos con una serie de proyectos que han utilizado distintos modelos de colaboración entre estos actores, desde los centralizados y cerrados hasta los abiertos y experimentales. Los modelos de interacción entre los actores, sus roles, resultados y la sustentabilidad de estos proyectos se constituirá como el principal ámbito de estudio.

El objetivo de este trabajo es analizar los distintos modelos de colaboración presentes en tres casos de iniciativas que han conectado diseñadores $y$ artesanos o productores. La metodología utilizada para el análisis comparativo es el estudio de casos, los dos primeros -Laboratorio de Diseño de Colombia y Rush Weaving de Taiwan- han sido elegidos por ser casos documentados y emblemáticos en la literatura disponible, incluyendo un tercero -SaberHacer de Chile-correspondiente al proyecto de uno de los autores de este articulo.

El aporte de este artículo se basa en identificar aquellos elementos en los modelos de colaboración que han impactado en la sustentabilidad de los proyectos estudiados y que podrían configurarse como insumos para la elaboración y sistematización de nuevos modelos de trabajo así como también de nuevas hipótesis que permitan generar nuevas investigaciones en este ámbito de estudio.

Palabras clave: Sistemas productivos, diseño, oficios, economía colaborativa, estudio de casos. 


\section{Introducción.}

Diversas iniciativas para la integración de diseñadores y artesanos o productores de manufactura han sido implementadas y documentadas al menos desde principios de la década de los noventa (Unesco, 2005), articulando trabajos en terreno apoyados por organizaciones internacionales, gobiernos locales, universidades y asociaciones de artesanos/productores, en búsqueda de una reivindicación del trabajo manual pero también de nuevas herramientas -en gran parte comerciales- que permitan a estos sistemas de producción ser sustentables en el tiempo. Sin embargo, el impacto de estas iniciativas parece ser mitigado por aspectos metodológicos internos y externos que no permiten la sostenibilidad, replicabilidad y/o escalabilidad de este tipo de proyectos.

Los motivos para la elaboración de esta investigación están relacionados al interés de aportar con nuevas estrategias de trabajo en este tipo de proyectos, observar cómo los aspectos colaborativos y la cultura open se encuentran o no presentes dentro de las iniciativas analizadas y cómo se relacionan a la metodología de trabajo y sus resultados.

En este artículo presentaremos un marco teórico del retorno de los oficios y una descripción de sus principales actores, analizaremos 3 casos de programas de vinculación entre diseñadores y artesanos/productores locales desde la perspectiva de la incorporación de estrategias colaborativas y open-source, observando sus principales resultados. La pregunta de investigación es: "¿Qué impacto tiene la estructura de actores del modelo colaborativo en la proyección a mediano y largo plazo del proyecto?". Finalmente se pretende identificar aquellos componentes y dinámicas que impactan en un buen resultado a mediano y largo plazo en cuanto a la creación de una comunidad de práctica.

\section{El retorno de los oficios y sus principales actores.}

Según el arquitecto y escritor Witold Rybczynski "el deseo de hacer algo bien, ya sea navegar en un velero o construirlo, refleja una necesidad que antes estaba relacionada con el lugar de trabajo" (1992, p. 202-203) pero en una época cada vez más automatizada y simplificada por la tecnología, el énfasis del desafío y profesionalismo de hacer algo difícil comienza a trasladarse al ocio, donde salir a trotar requiere de la vestimenta y los gadgets adecuados, con sistemas de medición de progreso y cumplimiento de metas. Rybczynski propone que el avance tecnológico ha terminado con la destreza en la mayoría de las profesiones, sea en la línea de montaje -donde hoy se necesita un mínimo de capacitacióny sobre todo en ambientes de prestación de servicios, en los que las habilidades sociales básicas son el requerimiento fundamental. 
Es así que el desafío del trabajo análogo de tiempos anteriores encuentra otros nichos donde emerger y las tendencias relativas al retorno de los oficios en la actualidad pueden ser explicadas a través de fenómenos sociales, laborales y económicos, donde la sobre-oferta profesional y la rutina del trabajo automatizado ha propiciado una revalorización del trabajo manual y el retorno de oficios, que permite a algunos profesionales guardar sus títulos en el cajón para dedicarse a ser zapateros, panaderos o carpinteros (Troncoso, 2014). Un punto importante que deja en evidencia esta situación es que en nuestra digitalizada y automatizada vida, muy pocos saben cómo se hacen las cosas que consumimos. Esta predisposición hacia el mundo material y el mercado que lo sustenta, parece ser una grieta en el continuo sentimiento de satisfacción hacia el constante avance tecnológico industrial actual.

\subsection{El consumidor.}

Un estudio sobre el deseo del consumidor de Sharon Zukin (2004), declaraba que el consumidor actual no tiene el conocimiento de producción que tenían las generaciones anteriores. "Hacia los años sesenta los norteamericanos ya no sabian ordeñar una vaca, cocinar una rosquilla ni hacer un coche con un envase de jabón o una caja de embalar" (Zukin, 2004, p. 185) y la evolución de este tipo de cultura del consumo rápido generó transformaciones radicales en cuanto a la forma como nos comportamos ante el consumo. Probablemente muchos de nuestros problemas actuales de sobreconsumo, desecho rápido de productos y su consecuente contaminación ambiental sean el efecto de un usuario que ya no entiende el producto que consume, de qué está hecho, cómo se fabrica, cómo se arregla y cómo se desecha (o recicla/reutiliza). Este cambio de mentalidad, que observamos en los distintos movimientos localistas, ambientalistas y contraculturales del capitalismo actual, apunta justamente al hecho de re-conocer el entorno industrial global desde la perspectiva de un consumidor mucho más informado de lo que compra, tal como lo plantea el movimiento Knowcosters ${ }^{1}$ de España, que busca promover el consumo consciente y las consecuencias de los actos del consumo, saber cuanto cuesta realmente un producto, dónde y cómo se fabrica, cuál es su valor económico, su costo para el planeta y el Estado de Bienestar.

Para el sociólogo Richard Sennett, "el consumidor moderno necesita pensar como un artesano sin la capacidad para hacer lo que hace el artesano" (Sennett, 2006, p.123) y esta forma comprar de un modo inteligente, con una experiencia basada en un relato social e histórico contenido en el objeto.

\footnotetext{
${ }^{1}$ http://www.knowcosters.org
} 


\subsection{El creador / diseñador}

Por otro lado, las tendencias relacionadas a los emprendimientos de la nueva generación de diseñadores de productos, que configuran su propuesta de valor desde una mirada mucho más consciente de los procesos productivos locales que pueden gatillar, son también un antecedente de esta necesidad de replantear la forma como consumimos y producimos bienes. Este auge del diseño basado en productos locales y de manufactura casi artesanal ha sido consecuencia muchas veces de crisis laborales, económicas o tendencias externas que han calado hondo en el quehacer de estos diseñadores que a veces colindan con la artesanía. Gui Bonsiepe describe al artesanato urbano como un modo de producción alternativo que surgió en crisis como la vivida por Argentina el año 2002, en la que diseñadores desempleados comenzaron a generar oferta de diseño local, para demanda local, con producción local y a precio local, inyectando nuevas dinámicas económicas que impactan en el territorio (Bonsiepe, 2010).

El Movimiento maker y el Slow Design, que describiremos a continuación, son antecedentes relevantes para comprender el auge del diseño basado en productos locales de manufactura casi artesanal.

\subsubsection{Movimiento Maker.}

El término es acuñado por Dale Dougherty, editor de tecnología que fundó la Make Magazine, revista enfocada al Do It Yourself (Hazlo tu mismo) y creador de las primeras Maker Faires en Estados Unidades. "De acuerdo al Media Lab del MIT, los makers tratan a los átomos como a los bits -utilizando las poderosas herramientas de la industria del software para revolucionar la forma en que hacemos objetos tangibles" (Hacedores.com, 2015). Lo relevante del concepto de Maker ha sido la puesta en valor de la fabricación en casa, de la capacidad de arreglar un objeto antes de tirarlo a la basura y comprar otro nuevo, usar las manos y ensuciarse por el solo hecho de mantener el control del conocimiento técnico del objeto que está en sus manos. Por supuesto, esto ha sido soportado principalmente por internet y la disponibilidad casi infinita de información y herramientas para construir un pequeño jardín en el departamento o arreglar la manilla de la puerta además de la masificación de tecnologías de fabricación digital -tales como la impresora $3 \mathrm{~d}$ y cortadoras láser por nombrar algunas- que han permitido la introducción de un nuevo formato de taller de trabajo y experimentación.

Chris Anderson comparaba el fenómeno de la disponibilidad de tecnología de fabricación digital de escritorio con la aparición de la primera impresora láser de escritorio, lanzada por 
Apple en la década de los ochenta, la cual permitió idear un nuevo escenario en que se pudo juntar el escritorio personal y el prototipado de una publicación, hecha en casa y con pocas unidades (Anderson, 2012).

\subsubsection{Movimiento Slow-design.}

La corriente del slow-design o 'diseño lento' tiene orígenes en el movimiento Slow y el Slow Food de Italia, que ha buscado recuperar una identidad local, de elaboración de alimentos en procesos lentos como contrapartida a la irrupción del fast-food norteamericano.

La aparición de este movimiento ha definido una serie de bajadas en distintos aspectos sociales y productivos que han planteado alternativas viables para 'rehumanizar' nuestro quehacer, volver a nuestras 'medidas' naturales de consumo y acciones locales concretas con las cuales contrarrestar los aspectos negativos de la globalización. El foco del Slow design está en el bienestar y este se encuentra integrado con una concepción del ser humano lento, con metabolismo acorde a un ritmo más lento de producción y consumo, entendiendo lo 'lento' como un valor sociocultural positivo, conectado con nuevos escenarios más sustentables (Grosse-Hering et. al, 2013).

El auge de estos nuevos emprendimientos de marcas de diseño local, derivados de distintas disciplinas, que han comenzado a desarrollar una conexión productiva más integrada con fabricantes y artesanos locales avanza, pero a tropiezos. La desindustrialización de los países occidentales y la interrupción del proceso de industrialización en muchos países de Latinoamérica impactó en la creciente escasez de productores locales así como también de estructuras organizativas y educativas en torno a la manufactura y los oficios, situación que evidencia los problemas en las cadenas de valor de diseñadores que producen localmente y la falta de capital productivo y asociativo de productores locales.

\subsection{El artesano/productor}

El productor, manufacturero local o artesano son sectores productivos que en mayor o menor medida han sido afectados por la integración de mercados vivida en las últimas décadas. La relocalización de la manufactura, en países con mano de obra barata -ubicados principalmente en el sudeste asiático- generó una serie de cambios en el sistema productivo local, desapareciendo gremios, empresas, talleres y estructuras educativas en oficios, tales como la Escuela de Artes y Oficios en Chile -fundada en 1849- que tenía como fin desarrollar nuevos artesanos y artífices para distintas áreas productivas (Castillo, 2010). Las consecuencias en el ámbito laboral, del cambio de políticas de desarrollo hacia adentro y de apertura de mercados, se pueden observar en los problemas laborales de los sectores más vulnerables, dada la baja diversificación de actividades económicas productivas y los 
bajos salarios que obtienen personas con oficios técnicos -artesanos, carpinteros, costureras, zapateros, etc- que ya no son valorados por empresas de servicios, principalmente orientadas a gestionar la comercialización de productos, fabricados globalmente (Riesco, 2012).

Dado este contexto actual, los productores/artesanos participan del mercado en condiciones adversas, con pocos gremios o asociaciones en los que apoyarse, reducidas estructuras educativas en relación a la educación técnicas y oficios de su rubro, bajo acceso a mejor tecnología para el desarrollo de su quehacer y un individualismo crónico que constituye una de las mayores brechas para generar nuevas redes entre productores y nuevos demandantes de producción.

\section{Metodología.}

Según Yin (1994, p. 13) el estudio de caso es:

"una investigación empírica que estudia un fenómeno contemporáneo dentro de su contexto de la vida real, especialmente cuando los límites entre el fenómeno y su contexto no son claramente evidentes. (...) Una investigación de estudio de caso trata exitosamente con una situación técnicamente distintiva en la cual hay muchas más variables de interés que datos observacionales; $y$, como resultado, se basa en múltiples fuentes de evidencia, con datos que deben converger en un estilo de triangulación; y, también como resultado, se beneficia del desarrollo previo de proposiciones teóricas que guían la recolección y el análisis de datos."

Las etapas de un estudio de caso son: 1) la definición de las preguntas de investigación ,2) la elección de los casos o las "unidades de análisis", 3) la recolección de data que se desarrolla en paralelo con el análisis y 4) la discusión (Baxter, 2008). Tal proceso investigativo aplicado a las iniciativas colaborativas permite indagar en profundidad acerca de las dinámicas observadas entre los distintos actores involucrados y las prácticas (métodos, herramientas, sistemas) que caracterizan la colaboración.

Para el presente análisis comparativo de casos elegimos proyectos colaborativos entre artesanos o pequeños manufactureros y diseñadores desarrollados en los últimos diez años en tres países. Dos proyectos son casos emblemáticos en la literatura, mientras que el tercero es un proyecto nuevo impulsado por uno de los autores de este artículo. En la recolección de data, ocupamos fuentes secundarias, dando de esta forma prioridad a los casos de estudio documentados en la academia. A continuación presentaremos los tres casos analizados, para finalmente seguir con una discusión y las conclusiones. 


\section{Casos de estudio.}

\subsection{Laboratorio de Diseño: Colección Casa Colombiana.}
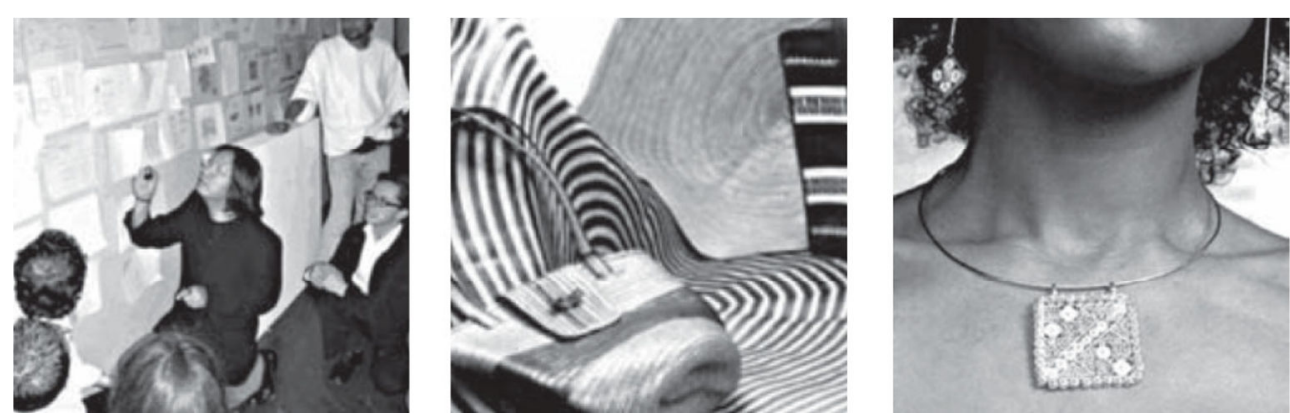

Fig. 1 Actividades y productos del proyecto Laboratorio de Diseño, Artesanias de Colombia.

\subsubsection{Descripción del Proyecto.}

El proyecto de Laboratorio de Diseño es gestado desde la Artesanías de Colombia, organización público-privada, vinculada al Ministerio de Comercio, Industria y Turismo del Gobierno Colombiano, que "contribuye al progreso del sector artesanal mediante el mejoramiento tecnológico, la investigación, el desarrollo de productos, y la capacitación del recurso humano, impulsando la comercialización de artesanías colombianas" (Artesanías de Colombia, 2016).

En el año 2001, se decide mejorar la calidad y los procesos productivos a través del diseño, el desarrollo de productos y la transferencia tecnológica, esto con el fín de impulsar los niveles de competitividad del sector, en un enfoque de exportación a mercados internacionales más exigentes (Unesco, 2005). Para la realización de este plan, se llevó a cabo un Proyecto de Asistencia Técnica Internacional que consistió en visitas de observación y diagnóstico a los laboratorios de diseño de Armenia y Pasto, visitas a comunidades y talleres artesanos en el país, evaluación de productos para artesanos interesados en participar en las Ferias Artesanales organizadas por la institución. Para ello se contrató al diseñador Filipino, Percy Jutare Arañador, quien lideró un proceso de capacitación y asistencia técnica a 50 diseñadores industriales, textiles, gráficos y arquitectos relacionados a los Laboratorios de Diseño de Armenia, Bogotá y Pasto. La capacitación también incluyó eventos masivos relacionados a temáticas de tendencias y marketing para el mercado internacional (Unesco, 2005). 


\subsubsection{Dinámicas de colaboración.}

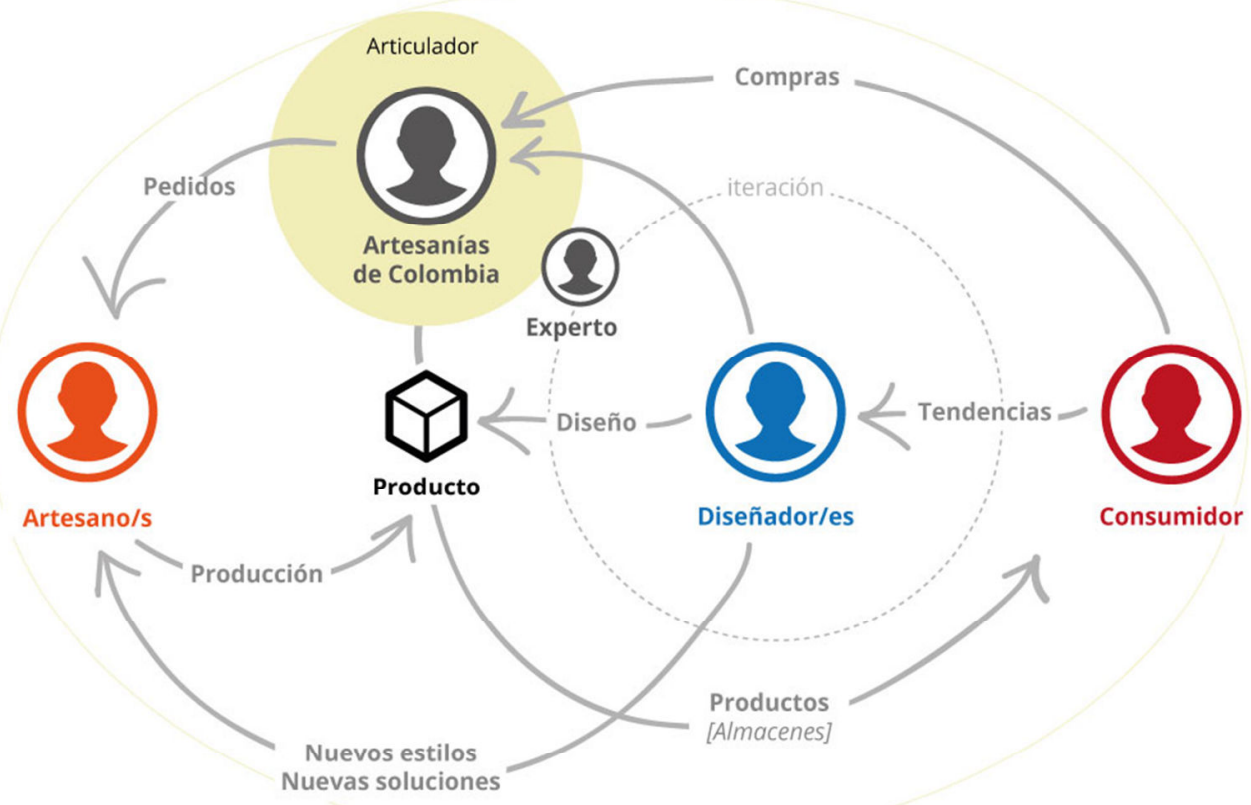

Fig. 2 Representación del modelo de colaboración proyecto "Laboratorio de Diseño"

El modelo de trabajo del proyecto Laboratorio de Diseño estaba basado en Comités de Trabajo con funciones específicas, liderados por un diseñador o coordinador, estos comités eran el Comité Creativo, Comité Técnico, Comité de Producción, Comité Comercial, Comité de Exhibición y Comité de Medios.

El trabajo con las comunidades de productores era definido desde el Laboratorio de Diseño, donde se establecía que perfiles y áreas de especialización de los talleres presentes concordaban con los productos a desarrollar, declarando la interactividad con los artesanos, respetando el saber tradicional de estos, sin embargo, el productor no participa en la generación de los productos.

Las herramientas de colaboración estaban basadas en seminarios de difusión de temas, mentoría por internet desde el experto a los diseñadores y el trabajo en terreno para la producción de productos. 
Estrategias colaborativas y open-source para la generación de sistemas productivos entre diseñadores y productores.

\subsubsection{Resultados.}

Los resultados de esta experiencia fue en primer lugar, la transferencia de conocimiento entre el experto internacional y diseñadores participantes de las distintas comisiones, quienes llevaron el conocimiento de tendencias hacia el desarrollo de productos que luego fue tangibilizado y encarrilado hacia comunidades de artesanos adhoc.

El segundo resultado fue el desarrollo del Proyecto Casa Colombiana cuyo propósito es "identificar y poner en valor una imagen con identidad nacional, a través del diseño y desarrollo de lineas de objetos funcionales y decorativos para un estilo de vida contemporáneo" (Unesco, 2015, p. 76)

El tercer resultado, relacionado con el anterior tiene que ver con las Colecciones desarrolladas durante el 2002 -basada en la utilización de materiales naturales locales-, el 2003 -basada en el café, como símbolo de identidad- y el 2004 -que conservaba el café como identidad, agregando elementos de la naturaleza local. Todas estas colecciones han sido exhibidas y comercializadas en la Casa Colombiana (Unesco, 2005).

\subsubsection{Sustentabilidad del Proyecto}

La dinámica de encuentro entre diseñadores y artesanos se encuentra inactivo, sin embargo, resultados tales como la Casa Colombiana y las colecciones desarrolladas durante el 2001 al 2004 aún siguen presentes en las estructuras comerciales de Artesanías de Colombia. Es posible que, la centralización de este proyecto en la gestión del articulador principal y las reducidas formas de transferencia de habilidades o capital productivo desde los diseñadores a los artesanos, no permitiera una mayor autonomía para la generación de emprendimientos $\mathrm{u}$ otras formas de replicabilidad del proyecto. 
4.2. Rush Weaving en Yuan-Li: Colaboraciones entre diseño y artesanía.
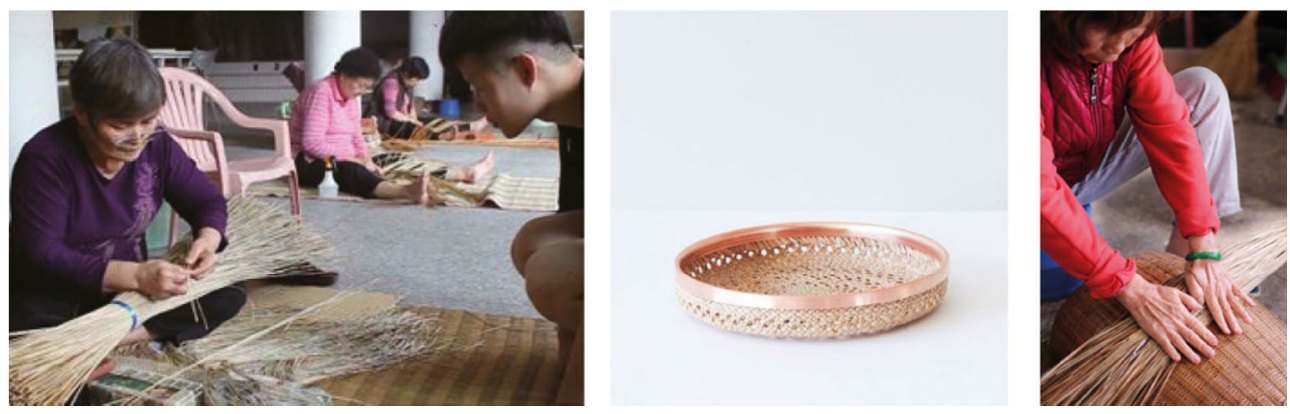

Fig. 3 Actividades y productos del proyecto Rush Weaving, Taiwan.

\subsubsection{Descripción del Proyecto.}

Yuan Li es una localidad de Taiwan con una tradición centenaria de actividad artesanal indígena, principalmente del trabajo en mimbre, con el cual se manufacturan esterillas, sombreros y carteras (Tung, 2012). Este sector ha sufrido una importante baja debido a la industrialización y la diversificación de alternativas en un amplio rango de estilos de diseño, con precios competitivos, convirtiendo la manufactura artesanal de estos productos en algo poco sustentable.

La transferencia de conocimiento de esta actividad ocurre en una enseñanza informal y a pesar de la habilidad de estos artesanos, su limitado manejo de técnicas no les ha permitido avanzar hacia productos más atractivos en el mercado actual.

El departamento de Industrial and Commercial Design del National Taiwan University of Science and Technology, la investigadora Fang-Wu Tung y estudiantes de diseño de la misma universidad llevaron a cabo una experiencia de trabajo en el año 2010 con una comunidad específica de artesanos tejedores de mimbre, esto con el fin de investigar la posibilidad de colaboración entre artesanos y diseñadores (Tung, 2012). 
Estrategias colaborativas y open-source para la generación de sistemas productivos entre diseñadores y productores.

\subsubsection{Dinámicas de colaboración.}

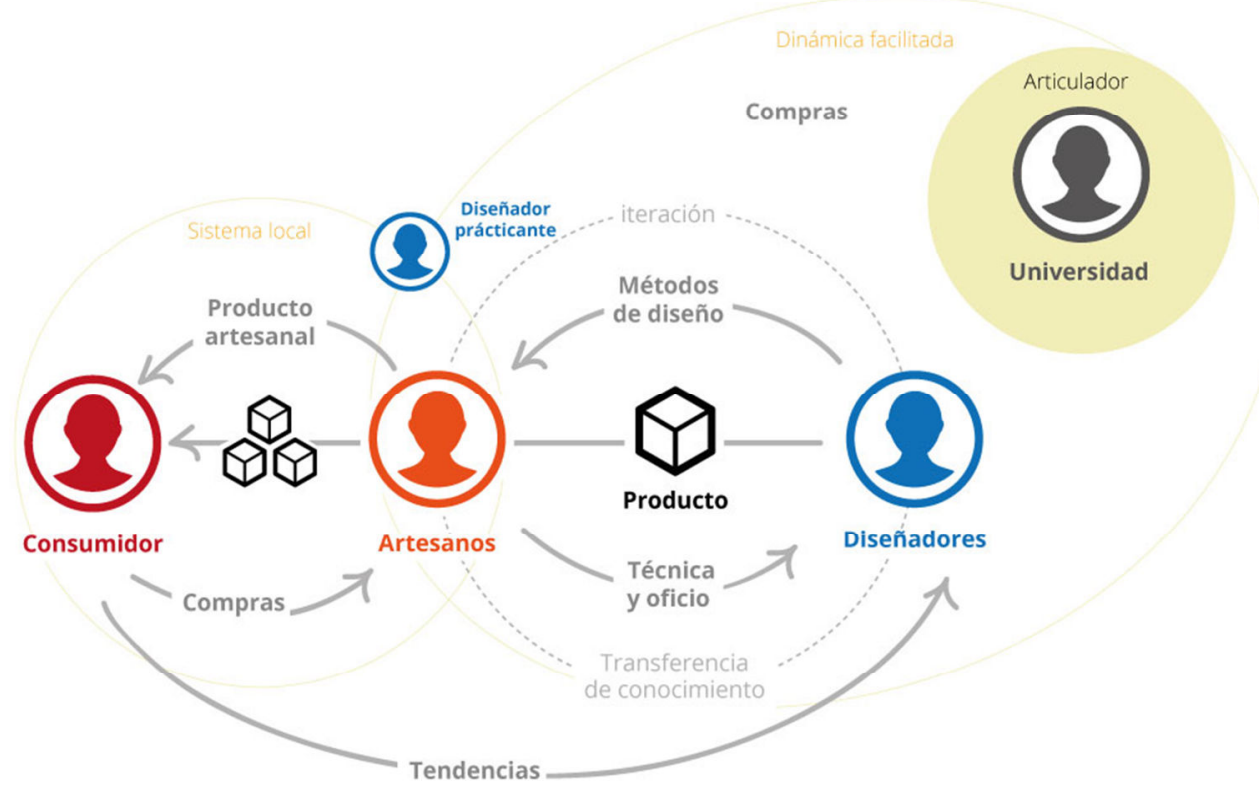

Fig. 4 Representación del modelo de colaboración proyecto "Rush weaving en Yuan-Li"

El modelo de trabajo que declara este proyecto se basa en una aproximación de co-creación, la cual se construye a partir de 3 grandes procesos:

Inicio difuso: Conociendo la localidad, visitando la comunidad artesanal y entrevistando a los actores claves que la movilizan y comercializan.

Diseño y desarrollo: (desarrollo de ideas, concepto, prototipado y productos con un foco colaborativo). El proceso de prototipado co-creativo permite habilitar a los artesanos en los métodos de producción y diseño, y los diseñadores pueden obtener una mejor comprensión del oficio y como fundir este con la modernidad.

Todo este proceso se encuentra entrelazado con discusión grupales, presentaciones e iteraciones que permiten obtener un prototipo ya testeado con los principales stakeholders. 
Transferencia del conocimiento y experiencia: El mecanismo de mutuo aprendizaje está basado en un intercambio de conocimiento de ambas partes para incrementar sus capacidades profesionales.

\subsubsection{Resultados.}

Como resultado del proyecto se desarrollaron dos líneas de productos basadas en Decoración de hogar que incluyeron lámparas, una radio y un taburete y accesorios personales basados en una serie de brazaletes, prototipos finales "que han servido como un vehículos para demostrar las posibilidades del tejido en mimbre para los stakeholders" (Tung, 2012, p. 79). Estos productos fueron exhibidos y lograron ganar varios premios, lo que aportó considerablemente a la visibilidad del proyecto y la comunidad de productores.

Por otro lado, al finalizar la experiencia un miembro del equipo de diseño se integró a la comunidad como practicante para aprender habilidades del oficio.

Esta iniciativa tuvo una trascendencia en la Universidad patrocinante, lo que permitió generar nuevos talleres anuales de diseño y artesanos.

\subsubsection{Sustentabilidad del Proyecto.}

A pesar de que se menciona la generación de nuevas iniciativas de talleres entre diseñadores y artesanos, en la actualidad no es posible localizar el proyecto. Esto nos lleva a pensar que la articulación de un proyecto de estas características con base 100\% académica, requiere de otras componentes para asegurar la proyección del mismo en ámbitos comerciales o productivos.

Sin embargo, el trabajo en mimbre de Yuan-Li si es posible de encontrar en exhibiciones internacionales tales como el London Craft Week 2016 (Native \& Co, 2016), con un énfasis en el diseño y la ancestralidad de este oficio manual. Este antecedente puede darnos pistas acerca del aporte del trabajo colaborativo y la transferencia de conocimiento mutuo de este proyecto, lo que permitió una proyección a nivel de emprendimientos no relacionados con la academia 
4.3. SaberHacer: Costureras + Diseñadores.
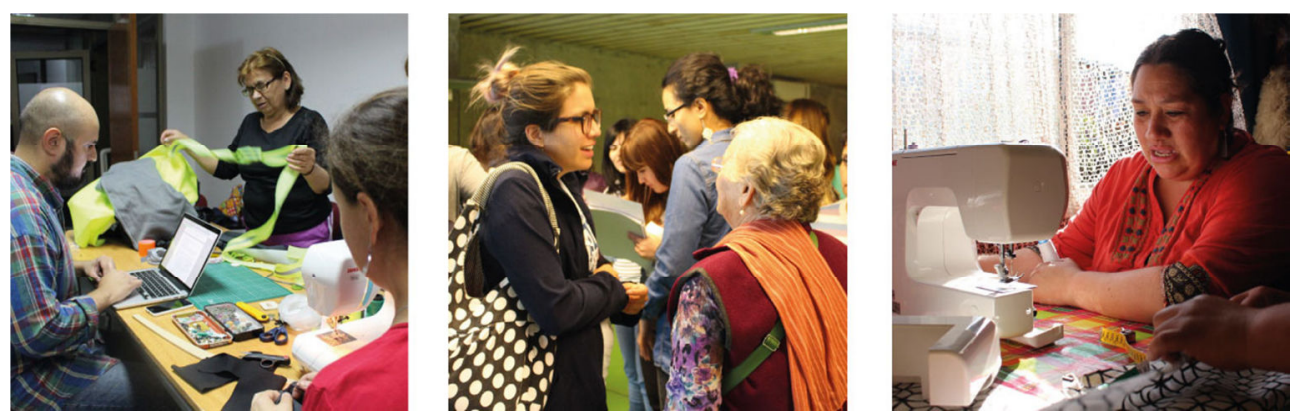

Fig. 5 Actividades del proyecto SaberHacer, Chile.

\subsubsection{Descripción del Proyecto.}

El proyecto SaberHacer fue iniciado por el diseñador Rodrigo Gajardo, en Santiago de Chile a partir del trabajo con la Municipalidad de Peñalolén y el área de Desarrollo Económico Local de la misma (Centro Yunus). Entre las distintas funciones de esta unidad municipal, se encuentra el apoyo a productores de la comuna en temas laborales, de emprendimiento y capacitación. Si bien, esta comuna de la ciudad de Santiago no presenta registros formales relevantes de actividad industrial, el sector de confección textil se constituía como un ámbito recurrente de atención, ya sea por programas de capacitación o apoyo comercial en emprendimientos de jefas de hogar que -a pesar de manejar diversas técnicas y disponer de tecnología adecuada para proveer al mercado- no logran mejorar sus ingresos y calidad de vida (Gajardo, 2015).

Como un modo de experimentar con nuevas metodologías de trabajo en este sector, se pilotearon una serie de actividades de vinculación entre costureras y diseñadores durante el año 2015, con el apoyo de la municipalidad y su infraestructura. 


\subsubsection{Dinámicas de colaboración.}

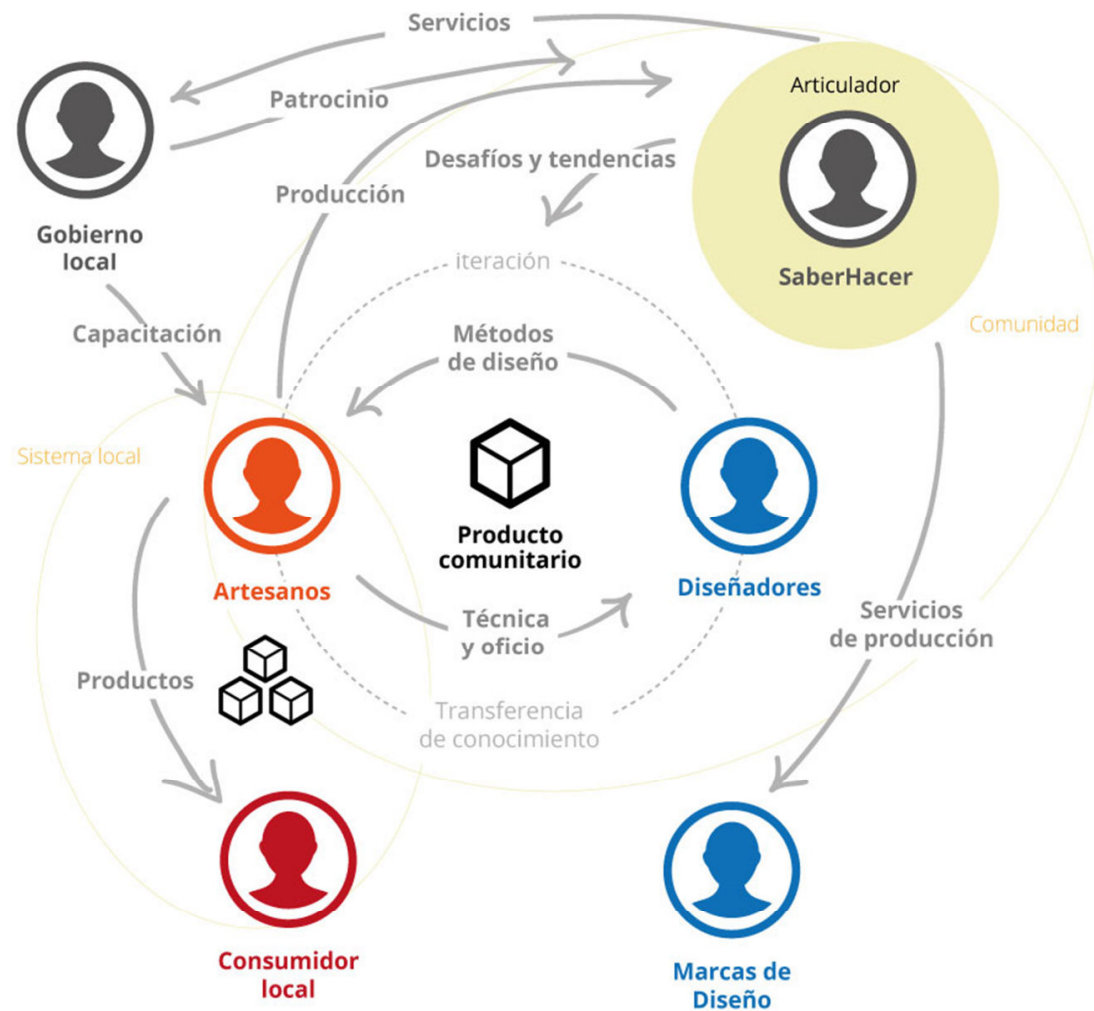

Fig. 6 Representación del modelo de colaboración proyecto "SaberHacer: Costureras + Diseñadores"

Los programas de SaberHacer han estado basados principalmente en el formato de Taller Colaborativo, cuyo foco está en reunir diseñadores y productores en 3 días de trabajo, desde el reconocimiento y conformación de equipos hasta el prototipado y liberación de conceptos de productos.

Día 1/ Trabajo colaborativo: Reconocimiento, conformación de equipos y entrega de desafíos.

Día 2/ Prototipado: Presentación de observaciones, referentes y prototipado \#1 de producto. Discusión entre pares. 
Día 3/ Producto y liberación: Presentación de mejoras, Prototipado \#2 y construcción de modelos de productos a liberar (planos, patrones, etc).

El proceso incluye espacios de transferencia cruzada de conocimiento, donde el diseñador puede aprender nuevas técnicas para trabajar materialidades (ej: cortar una circunferencia en tela, utilizar una máquina de coser, uso de herramientas, etc) y los productores/artesanos aprenden métodos de diseño para la generación de nuevos productos (ej: observación, búsqueda de referentes, manejo formal, procesos de iteración, etc).

Al cierre de estas actividades cada participante es ingresado formalmente a la Comunidad de Productores locales, con la entrega de una credencial con acceso al sitio web y al portafolio de productos comunitarios.

\subsubsection{Resultados.}

Al término de las actividades desarrolladas durante el año 2015 y luego de trabajar con casi 40 costureras y 25 diseñadores en dos instancias de Talleres colaborativos se obtuvieron una serie de resultados que se han configurado como el Capital productivo de la comunidad.

Un primer resultado fue la generación de 21 prototipos de productos abiertos, resultantes de los distintos talleres aplicados -entre los que encontramos productos para ciclistas, hogar, mascotas, runners, entre otros- los cuales se encuentran disponibles en el sitio web del proyecto donde los miembros de la comunidad pueden 'descargar' los patrones constructivos para su uso, fabricación y comercialización.

El segundo resultado fue la generación de una Comunidad de Productores Locales, que es el resultado de aquellas personas que han participado de las actividades, quienes mantienen una relación de apoyo y generación de servicios (ej: diseñadores que contratan costureras, costureras que se asociación para un trabajo específico).

El tercer resultado del proyecto ha sido la experiencia de una serie de proyectos comerciales gestionados por la organización articuladora, donde se ha vinculado a un grupo de productores con 2 marcas de diseño local que han requerido servicios de corte y confección para sus líneas de productos. En otro caso, el grupo de diseñadores y costureras han desarrollado un proyecto interno, desde la ideación hasta la producción de servilletas y manteles de tela estampados en serigrafía artesanal (SaberHacer, 2016). 


\subsubsection{Sustentabilidad del Proyecto}

Si de alguna forma este proyecto reúne las características colaborativas de transferencia de conocimiento mutuo entre diseñadores y productores del proyecto taiwanes y la centralidad de la gestión del proyecto colombiano, los desafíos para encontrar una sustentabilidad están justamente en la complejidad observadas de las interacciones y gestiones requeridas para movilizar a todos los actores involucrados.

\section{Discusión.}

En la selección de casos, privilegiamos la diversidad de los modelos de colaboración en cuanto a articulaciones que se dan entre los iniciadores del proyecto y los actores participantes, por sobre la cantidad de casos. Los resultados que nos arroja este análisis comparativo nos da luces acerca de cómo se comportan las distintas iniciativas en el tiempo según el tipo de modelo de colaboración que se aplique y cómo el enfoque abierto o cerrado de la transferencia de conocimientos tiene un impacto en la sustentabilidad o replicabilidad de los mismos.

Si bien no es posible, por el tamaño de la muestra, demostrar una relación de causalidad entre el modelo de colaboración y el impacto generado en la sustentabilidad de los proyectos, si hemos obtenido algunas hipótesis de la correspondencia entre los modelos y la trayectoria de los proyectos en el tiempo.

Algunas de estas hipótesis tienen relación a cómo la centralidad cerrada de estas iniciativas afecta la generación de nuevas iniciativas desde los actores involucrados, cuánto afecta el academicismo puro en la generación de modelos de negocios y relaciones comerciales concretas más allá de la experimentación así como también los desafíos de gestión requeridos cuando ambos mundos -el académico y el comercial/productivo- intentan fundirse en una misma iniciativa.

Estas hipótesis se deberían validar en un trabajo futuro analizando una muestra mayor de casos, a través de un mapeo sistemático o búsqueda sistemática.

\section{Conclusiones.}

El enfoque estratégico del diseño, que hasta ahora ha sido utilizado para desarrollar de manera sistémica y sistemática las ventajas competitivas de las empresas -articulando sus sistemas-producto-servicio- hoy tiene la oportunidad de operar en nuevas lógicas. Gracias a enfoques tales como el diseño de servicios y la continua sistematización de herramientas 
para este quehacer, el diseñador estratégico puede incorporarse a los desafíos que sugiere el sistema público y las comunidades, basándose en una mirada holística del contexto, resolviendo problemáticas desde su mirada centrada en las personas y ubicando su trabajo en esa intersección generada entre la tecnología, la técnica, el mercado y las personas, pasando de medir su efectividad no sólo desde la rentabilidad económica o efectividad técnica sino también desde la rentabilidad social. Este foco, en el contexto de la producción local y la generación de economías locales hace sumamente interesante la intervención del diseño, no sólo desde su dimensión estratégica sino que también desde las distintas especialidades que pueden relacionarse con el quehacer de artesanos, manufactureros y productores locales.

El requerimiento de diseñar nuevos sistemas productivos que permitan articular a los distintos actores involucrados en estos nuevos escenarios locales incluye repensar modelos de negocios, estrategias de capacitación y generación de capital productivo en economías colaborativas. La estrategia del uso de talleres colaborativos en la generación de soluciones compartidas entre diseñadores y productores, así como también la incorporación de estrategias open source en el uso libre de este capital por cualquier actor del sistema, podría ayudar en la efectiva generación de nuevos sistemas productivos que atiendan nuevos mercados sustentables.

\section{Referencias}

ANDERSON, C. (2012). "Makers, The New Industrial Revolution". New York: Crown Business.

BAXTER, P., Jack, S. (2008). Qualitative Case Study Methodology: Study Design and Implementation for Novice Researchers. The Qualitative Report, vol.13, número 4, pp. 544559.

BONSIEPE, G. (2010). El diseño en tiempos de turbulencias, $1^{\circ}$ Congreso Internacional de Diseño e Innovación de Cataluña, Escuela Superior de Diseño ESDI. 18 y 19 de Marzo de 2010 .

GROSSE-HERING, B., MASON, J., ALIAKSEYEU, D., BAKKER, C., DESMET, P. (2013). "Slow Design for meaningful interactions" en Proceedings of the SIGCHI Conference on Human Factors in Computing Systems. Paris. Disponible en $<$ https://www.researchgate.net/publication/262313813_Slow_Design_for_meaningful_inter actions $>$ [Consulta: 4 de mayo de 2016]

GAJARDO, R. (2015). "SaberHacer: Plataforma de conocimiento productivo basado en los oficios, la manufactura y el diseño de productos para el desarrollo económico local" (Tesis de magister). Pontificia Universidad Católica de Chile. Santiago de Chile.

HESS, D. (2009). Localist Movements in a Global Economy. Sustainability, Justice, and Urban Development in the United States. Cambridge: The MIT Press.

NATIVE \& CO. Lin: The Art of Taiwanese Rush Weaving. $<$ http://www.nativeandco.com/journal/lin-the-art-of-taiwanese-rush-weaving $>$ [Consulta: 2 de mayo de 2016] 
RIESCO, M. (2012) Mal Holandés. <http://economia.manuelriesco.cl> [Consulta: 5 de Enero de 2016]

RYBCZYNSKI, W (1992). Esperando el fin de semana. Barcelona: Emecé Editores.

SENNETT, R. (2009). El artesano. Barcelona: Editorial Anagrama.

SABERHACER: Vuelve a la tela. $<$ http://www.saberhacer.cl/vuelve-a-la-tela $>$ [Consulta: 11 de mayo de 2016]

TRONCOSO, P. (2014) "La vuelta de los oficios." Santiago de Chile: La Tercera. $<$ http://www.latercera.com/noticia/tendencias/2014/11/659-602699-9-la-vuelta-de-losoficios.shtml> [Consulta: 2 de mayo 2016]

TUNG, F. W. (2012). Exploring Craft-Design Collaborations in Revitalizing a Local Craft, $\begin{array}{llllll}\text { International Journal of Design } & \text { Vol.6 } & \text { No.3 } & \text { 2012, }\end{array}$ $<$ http://www.ijdesign.org/ojs/index.php/IJDesign/article/view/1077> [Consulta: 24 de marzo de 2016]

UNESCO. (2005). "Encuentro entre Diseñadores y Artesanos, Guía Práctica". Nueva Delhi: Craft Revival Trust, Artesanías de Colombia S.A. y UNESCO.

YIN, R. K. (1994). Case Study Research: Design and Methods. Sage Publications, Thousand Oaks, CA.

ZUKIN, S. (2004). Point of Purchase. Londres: Routledge. 